\title{
Plasma Histone H4 and H4K20 Trimethylation Levels Differ Between Colon Cancer and Precancerous Polyps
}

\author{
EMRE ÖZGÜR $^{1}$, METIN KESKIN $^{2}$, EBRU E. YÖRÜKER ${ }^{1}$, STEFAN HOLDENRIEDER $^{3}$ and UGUR GEZER ${ }^{1}$ \\ ${ }^{1}$ Department of Basic Oncology at Oncology Institute, Istanbul University, Istanbul, Turkey; \\ ${ }^{2}$ Department of General Surgery, Istanbul Medical Faculty, Istanbul University, Istanbul, Turkey; \\ ${ }^{3}$ Department of Laboratory Medicine, German Heart Center at Munich Technical University, Munich, Germany
}

\begin{abstract}
Background/Aim: No blood-based biomarkers are available to differentiate between colonic tumors and precancerous polyps. Previously we demonstrated levels of trimethylated H4K2O (H4K2Ome3) to be lower in blood plasma from patients with colon cancer than those from cancer-free individuals. Herein, we added individuals with precancerous polyps for the first time in order to analyze and investigate the usefulness of plasma H4K2Ome3 and histone $\mathrm{H} 4$ to discriminate colon tumors from precancerous polyps. Materials and Methods: The study included a cohort of 185 individuals undergoing colonoscopy. H4K20me3 and histone H4, measured by an enzyme-linked immunosorbent assaylike assay in plasma, were analyzed according to colonoscopy findings. Results: Levels of H4K2Ome3 were lower in patients with colon cancer than in individuals with normal colonoscopy and those with precancerous polyps $(p=0.02$ and $p=0.01$, respectively). In contrast, highest quantities of histone $\mathrm{H} 4$ were measured in those with colon cancer compared to other groups (all $p<0.01$ ). Conclusion: Beside H4K2Ome3, plasma histone H4 is a useful marker to discriminate colonic tumors from precancerous polyps and other conditions.
\end{abstract}

Colon cancer is the most common malignancy of the gastrointestinal tract and the third leading cause of cancerrelated death, with a decline in the incidence and mortality since 1998 (1). Only a small proportion (approx. 14\%) of colon cancer cases are diagnosed at an early stage. Overall survival rates are higher (around $90 \%$ at 5 years) for patients

This article is freely accessible online.

Correspondence to: Dr. Ugur Gezer, Istanbul University, Oncology Institute, Department of Basic Oncology, 34093 Capa, Istanbul, Turkey. E-mail: ugurd@istanbul.edu.tr

Key Words: Colon cancer, precancerous polyps, blood plasma, histones, histone methylation. with cancer localized to the colon. Accordingly, survival declines with the advancement of disease, being only $14 \%$ at 5 years after diagnosis for patients with distant metastases (2).

Colon cancer typically develops from precancerous polyps, mainly of adenomatous pattern (3). Thus, screening and detection of early-stage precancerous polyps in averagerisk individuals make colon cancer a preventable disease and accordingly has led to substantial reduction in the incidence of colon cancer. Each with different strengths and limitations, multiple screening options such as stool-based tests, flexible sigmoidoscopy, and colonoscopy are available (4). Among direct visualization tests, colonoscopy has proven the most effective examination for reducing colon cancer mortality (5) and is considered 'gold standard' in colon cancer screening. However, it is an invasive procedure, affecting patient compliance, and is associated with some risks, including bleeding.

Most colonic/rectal tumors develop through a multistep process involving a series of histological, morphological, and genetic/epigenetic alterations that accumulate over time (6). The underlying genetic/epigenetic mechanisms in the development of colon cancer are quite heterogeneous (7). This heterogeneity results in a lack of reliable biomarkers with high sensitivity and specificity for detection of colonic tumors. In recent years, liquid biopsy has increasingly been recognized as a novel source of biomarkers (8). Several candidate biomarkers are being tested for their relevance and potential utility in cancer, including colon cancer. A potential field of blood-based biomarkers is the detection of cancer-related histone modifications in blood. This is a rapidly growing area of biomarker research (9-13). By employing chromatin immunoprecipitation (ChIP)-based polymerase chain reaction (PCR) and the sequencing of ChIP-related plasma DNA or simple enzyme-linked immunosorbent assay (ELISA)-like measurement, we previously showed levels of trimethyl histone H3 lysine 20 (H4K20me3), a heterochromatinenriched modification, to be lower in plasma samples from patients with colonic/rectal cancer than in plasma from cancerfree individuals (9-11). 
In this cross-sectional study, we assessed the relevance of plasma H4K20me3 and histone $\mathrm{H} 4$ in discriminating colonic cancer from precancerous polyps or other conditions. We added total histone $\mathrm{H} 4$ as an additional marker to the analysis. Individuals with precancerous polyps were added for the first time as a control group to the analysis.

\section{Materials and Methods}

Study subjects. The study cohort included 185 individuals who underwent colonoscopy at the Department of Surgery at Istanbul Medical Faculty Hospital for various reasons between September 2016, and June 2018. The study variables (H4K20me3 and total H4) were measured with no knowledge of the colonoscopy outcomes. After measurement of the samples, the provided colonoscopy findings were as follows: normal colonoscopy findings in 79 , diverticular disease in 15 , non-neoplastic polyps in 15 , neoplastic/precancerous polyps in 31 , colon tumors in 40 , and other findings in four (Table I). Diagnosis of colonic polyps and colonic tumors was made pathologically from biopsy specimens taken during colonoscopies. This study was approved by the Ethics Committee of Istanbul Medical Faculty (approval number 2015/413128), and informed consent was obtained from the participants.

Processing blood samples. Blood samples were taken into EDTAtubes before the colonoscopy procedure and within $3 \mathrm{~h}$ of withdrawal were centrifuged $(10 \mathrm{~min}$ at $717 \times \mathrm{g})$ to obtain the plasma fraction, which was then re-centrifuged and stored in aliquots at $-80^{\circ} \mathrm{C}$ until assayed.

Measurement of H4K2Ome 3 and total histone H4 in blood plasma. The commercially available EpiQuik Global Tri-Methyl Histone Quantification Kit (Epigentek, Farmingdale, NY, USA) and the EpiQuik Total Histone H4 Quantification Kit (Epigentek) were employed for the ELISA-like measurement of H4K20me3 and total histone $\mathrm{H} 4$ in plasma, respectively. Intra- and intra-assay variability analysis and measurements were determined as described previously (11). The intensity of absorbance was proportional to histone methylation or total histone $\mathrm{H} 4$ concentration in plasma. Mean absorbance values from duplicates were considered relative plasma levels of H4K20me3 or histone H4.

Quantitation of H4-linked nucleosomal DNA. As histone H4 measurement was included for the first time in our plasma histone research, we wished to quantify H4-related nucleosomal DNA in patients with colon cancer and precancerous polyps. For this purpose, nucleosomal DNA was extracted from nucleosomes that bound to anti-H4 antibody immobilized on assay wells. For this, 50 $\mu l$ plasma was added to assay wells and after antigen-antibody binding, wells were washed several times. Nucleosomal DNA was released from immune complexes by adding $100 \mathrm{mM} \mathrm{NaHCO}_{3}, 1 \%$ sodium dodecyl sulfate and proteinase $\mathrm{K}$ and incubating plates at $65^{\circ} \mathrm{C}$ for $15 \mathrm{~min}$. Subsequently, extracted DNA was cleaned using an appropriate DNA purification kit (Roche Diagnostics, Mannheim, Germany). Nucleosomal DNA was then quantified by amplifying the long interspersed element-1 (LINE1) repeat in quantitative PCR using SYBR Green (Power SYBR Green Master Mix; Applied Biosystems, Austin, TX, USA) in LightCycler 480 Instrument (Roche Diagnostics). By serial dilution of genomic DNA with
Table I. Colonoscopy findings of the study participants.

\begin{tabular}{lrrrr}
\hline & & \multicolumn{2}{c}{ Gender, $\mathrm{n}$} & \\
\cline { 3 - 4 } Colonoscopy finding & $\mathrm{n}$ & Female & Male & $\begin{array}{c}\text { Median age, } \\
\text { years }\end{array}$ \\
\hline Normal colonoscopy & 79 & 49 & 30 & 53 \\
Colonic polyps & 46 & 19 & 27 & 59 \\
Neoplastic & 31 & & & \\
Non-neoplastic & 15 & & & \\
Colonic tumor & 40 & 18 & 22 & 61 \\
Diverticular disease & 15 & 5 & 10 & 65 \\
Other & 4 & 2 & 2 & 58 \\
\hline
\end{tabular}

known concentration, a standard curve was generated, and the concentration of nuclesomal DNA was determined in $\mathrm{ng} / \mathrm{ml}$.

Measurement of carcinoembryonic antigen (CEA) and cancer antigen 19-9 (CA19-9) in patients with colon cancer. CEA and CA19-9 levels were measured in sera of patients with colon cancer using commercial assays (Roche Diagnostics) that were run on an immunochemistry analyzer (COBAS E411; Roche Diagnostics). Measurement of the tumor markers was based on an electrochemiluminescence sandwich immunoassay. The lower limit of CEA detection was $0.2 \mathrm{ng} / \mathrm{ml}$ and that of CA19-9 was $0.6 \mathrm{U} / \mathrm{ml}$.

Statistical analysis. We present study findings as median, quartiles and total ranges. Discrimination between the study groups was assessed using the Mann-Whitney $U$-test. Sensitivity and specificity profiles for all possible cutoffs of plasma H4K20me3 and histone H4 were assessed using receiver operating characteristic (ROC) curves and areas under the curves (AUC). A $p$-value of less than 0.05 was considered statistically significant. Calculations were performed using the statistical software GraphPad Prism 5 (GraphPad Inc., La Jolla, CA, USA).

\section{Results}

Plasma H4K20me3, and histone H4 levels were measured for 177 individuals in a blinded manner irrespective of colonoscopy finding. We found no correlation between H4K20me3 and total histone H4 in plasma (data not shown). After measurement, values were assessed according to colonoscopy findings. Figure 1A illustrates plasma levels of H4K20me3 and H4 in individuals with normal colonoscopy findings, patients with diverticular disease, non-neoplastic polyps, precancerous polyps, and colonic/rectal tumors. We found the lowest levels of H4K20me3 in plasma from patients with colonic cancer (relative median value 0.19 ). In other groups, relative plasma values of H4K20me3 ranged from 0.23 to 0.29 . The difference between patients with colon cancer and individuals with normal colonoscopy findings or neoplastic polyps was statistically significant ( $p=0.02$ and $p=0.01$, respectively). This finding is in line 

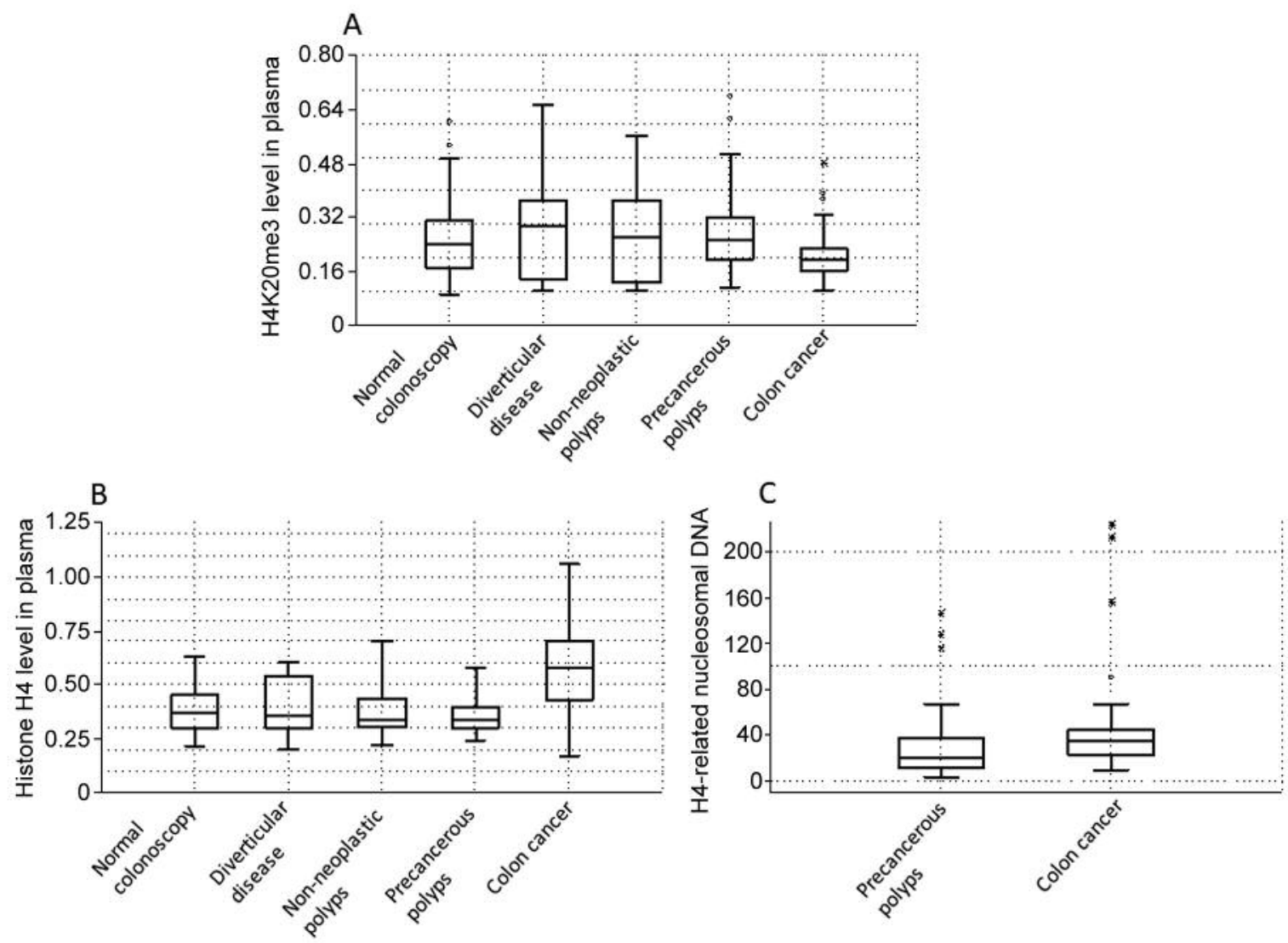

Figure 1. Plasma level of histone H4K20me (A), H4 (B) and H4-related nucleosomal DNA (C). Commercially available kits were employed to measure plasma $\mathrm{H} 4$ and H4K2Ome3 levels by an enzyme-linked immunosorbent assay-like approach using 50 ul of plasma samples in duplicate. The intensity of absorbance is proportional to total histone H4 or H4K2Ome3 concentration. Mean absorbance values were considered relative plasma levels of H4K2Ome3 or histone H4. Nucleosomal DNA was extracted from anti-H4 antibody-bound nucleosomes and was quantified in qPCR by amplifying the long interspersed element-1 (LINE1) element. The amount of DNA is shown in $\mathrm{ng} / \mathrm{ml}$. The bars indicate the ranges of the measured parameter. The lines in boxes represent median values, whereas the boxes symbolize the 25-75\% percentiles. Asterisks indicate extreme values.

with our previous reports (9-11) confirming lower levels of H4K20me3 in blood circulation of patients with colon cancer and reveals that plasma H4K20me3 levels differ between colon cancer and precancerous states.

In contrast to H4K20me3, we found markedly higher quantities of total histone $\mathrm{H} 4$ in plasma from patients with colon cancer compared with other groups (Figure 1B). The relative median level of total $\mathrm{H} 4$ in patients with colon cancer (0.57) was significantly higher than that in individuals with normal colonoscopy $(0.37, p<0.001)$, patients with diverticular disease $(0.35, p=0.002)$, non-neoplastic polyps $(0.34, p=0.008)$ or with precancerous polyps $(0.34$, $p<0.001)$. This marked difference in $\mathrm{H} 4$ histone level was also documented by quantifying H4-related nucleosomal DNA. This analysis revealed significantly higher levels of H4-related nuclesomal in patients with colon cancer compared with those with precancerous polyps (median 36 vs. $20 \mathrm{ng} / \mathrm{ml}$, respectively) (Figure 1C, $p=0.02$ ).
The ROC curves in Figures 2 and 3 exemplify the capability of plasma H4K20me3 and histone $\mathrm{H} 4$ to discriminate those with colon cancer from those with other conditions. For plasma H4K20me3, we obtained AUC values of $61 \%$ and $69 \%$ for discriminating colonic tumors from normal colonoscopy findings and precancerous polyps, respectively. The diagnostic potential of histone $\mathrm{H} 4$ was higher, with AUC values of $79 \%, 83 \%$ and $79 \%$, for discriminating colon cancer from normal colonoscopy and precancerous polyps, and all non-cancer conditions, respectively. The median value of histone $\mathrm{H} 4$ (0.57) in patients with colon cancer had $50 \%$ sensitivity and $91 \%$ specificity for discriminating them from individuals with normal colonoscopy. The specificity of this value was higher (96\%) for the discrimination of colon cancer from precancerous polyps.

Finally, we analyzed the association of H4K20me3 and histone $\mathrm{H} 4$ with clinical characteristics of the patients with 

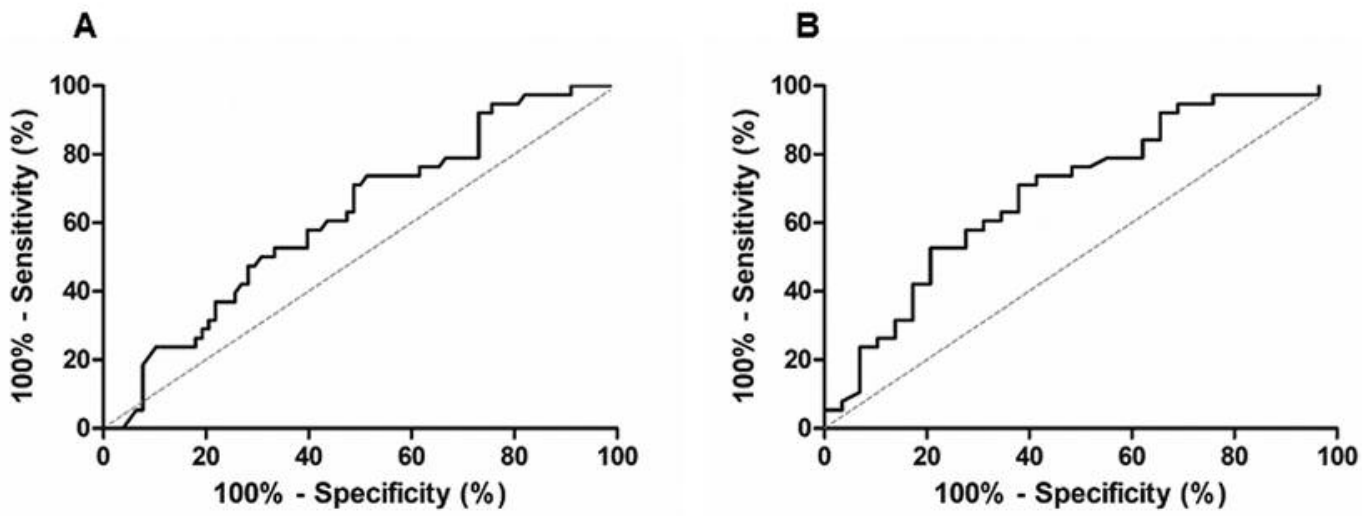

Figure 2. Diagnostic value of plasma H4K20me3 in colon cancer. Receiver operating characteristic curves were generated comparing patients with colon cancer and individuals with normal colonoscopy findings $(A)$ and those with precancerous polyps (B), with area under the curve values of $61 \%$ and $69 \%$, respectively.

Table II. Association of H4K2Ome3 and histone $\mathrm{H} 4$ with demographic and clinical characteristic of patients with colon cancer.

\begin{tabular}{|c|c|c|c|c|c|c|}
\hline Variable & & $\begin{array}{c}\text { Number of } \\
\text { patients }\end{array}$ & $\begin{array}{c}\text { Median H4K20m3 } \\
\text { level }\end{array}$ & $p$-Value & $\begin{array}{c}\text { Median histone H4 } \\
\text { level }\end{array}$ & $p$-Value \\
\hline \multirow[t]{2}{*}{ Age } & $\geq 60$ Years & 22 & 0.18 & 0.8 & 0.58 & 0.7 \\
\hline & $<60$ Years & 18 & 0.19 & & 0.57 & \\
\hline \multirow[t]{2}{*}{ Gender } & Male & 22 & 0.18 & 0.4 & 0.52 & 0.1 \\
\hline & Female & 18 & 0.21 & & 0.60 & \\
\hline \multirow[t]{2}{*}{ Tumor diameter } & $\geq 5 \mathrm{~cm}$ & 25 & 0.19 & 0.6 & 0.59 & 0.8 \\
\hline & $<5 \mathrm{~cm}$ & 15 & 0.18 & & 0.54 & \\
\hline \multirow[t]{2}{*}{ Tumor localization } & Left colon & 20 & 0.18 & 0.7 & 0.58 & 1.0 \\
\hline & Right colon & 20 & 0.19 & & 0.57 & \\
\hline \multirow[t]{2}{*}{ Diferentiation } & Well/medium & 29 & 0.22 & 0.1 & 0.54 & 0.2 \\
\hline & Poor & 11 & 0.13 & & 0.64 & \\
\hline \multirow[t]{2}{*}{ Lymphatic metastasis } & No & 17 & 0.14 & 0.02 & 0.60 & 0.9 \\
\hline & $\mathrm{N} 1, \mathrm{~N} 2, \mathrm{~N} 3$ & 23 & 0.31 & & 0.55 & \\
\hline \multirow[t]{2}{*}{ Lymphocyte infiltration } & No & 27 & 0.18 & 0.4 & 0.59 & 0.1 \\
\hline & Yes & 13 & 0.21 & & 0.51 & \\
\hline \multirow[t]{2}{*}{ CEA } & $\leq 5 \mathrm{ng} / \mathrm{ml}$ & 26 & 0.18 & 0.1 & 0.52 & 0.2 \\
\hline & $>5 \mathrm{ng} / \mathrm{ml}$ & 14 & 0.14 & & 0.64 & \\
\hline \multirow[t]{2}{*}{ CA19-9 } & $\leq 37 \mathrm{U} / \mathrm{ml}$ & 32 & 0.23 & 0.8 & 0.58 & 0.8 \\
\hline & $>37 \mathrm{U} / \mathrm{ml}$ & 8 & 0.21 & & 0.56 & \\
\hline
\end{tabular}

cancer, including tumor markers CEA and CA19-9 (Table II). This analysis revealed plasma H4K20me3 to be associated with lymphatic metastasis as patients with positive lymph nodes had significantly higher levels of H4K20me3 than those with N0 tumors. For histone H4, we found no significant associations.

\section{Discussion}

Using different techniques, we previously showed plasma levels of H4K20me3 to be lower in patients with colon cancer than in healthy individuals $(9,10)$. In the most recent study, we employed colonoscopy-verified colonic cancer-free individuals without colonic polyps as a control group (11). Here in addition to colonoscopy-verified colon cancer-free individuals, patients with precancerous polyps were included in the study cohort as controls. From a cohort of 177 individuals who underwent colonoscopy, 29 individuals were found to have precancerous polyps. In addition to H4K20me3, we included total histone $\mathrm{H} 4$ for the first time in the analysis.

Our results confirm the level of H4K20me3 in blood plasma to be lower in patients with colon cancer than in control groups, including those individuals with precancerous polyps. This finding argues for tumor specificity in the 

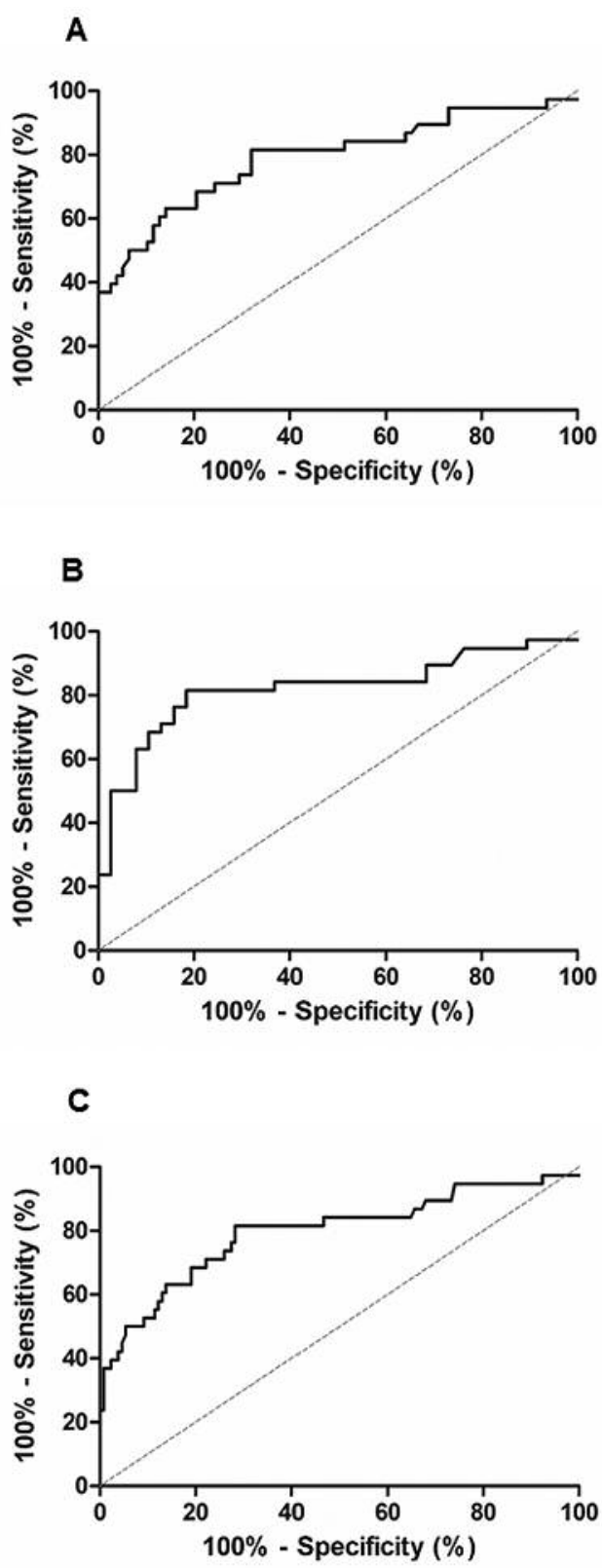

Figure 3. Diagnostic value of plasma histone $\mathrm{H} 4$ in colon cancer. Receiver operating characteristic curves include the comparison of patients with colon cancer with individuals with normal colonoscopy findings $(A)$, those with precancerous polyps $(B)$, and all non-cancer $(C)$, with area under the curve values of 79\%, $83 \%$, and $79 \%$, respectively.

reduction of H4K20me 3 in blood circulation and in line with previous reports describing global loss of H4K20me3 in cancer cells (14-17). However, due to the high heterogeneity possible amongst colonic tumors, the diagnostic potential of this marker appears to be limited. Not only tumor heterogeneity, but also stability issues of histone methylation in blood circulation might be confounding factors in variation of H4K20me3 levels among patients with cancer and controls. On the other hand, colonoscopy and many pre-analytical variables were shown to have no significant influence on the level of histone methylation in circulating nucleosomes (18).

We found higher quantities of total histone $\mathrm{H} 4$ in blood plasma of patients with colon cancer than in control groups. More interestingly, the statistical power of total histone $\mathrm{H} 4$ in discriminating colon cancer from precancerous polyps or other conditions was higher than that of H4K20me3. Subgroup analysis revealed no difference between small and larger tumors, suggesting diagnostic applicability of this marker in all stages. Elevation of total histone $\mathrm{H} 4$ in blood plasma of patients with colon cancer is likely a result of increased release of nucleosomes into the circulation as circulating nucleosomes are detected at increased rates in patients with cancer due to higher cellular turnover (19) and are relatively stable in circulation (20). In accordance with this, we found higher levels of histone H4-related nucleosomal DNA in patients with colon cancer than in those with precancerous polyps, confirming higher quantities of nucleosomes in colon cancer. However, this was not reflected in plasma levels of H4K20me3, indicating that total amounts of histones are not an index of histone methylation levels in blood circulation.

In summary, in line with our previous reports, the findings of the present study indicate a reduction of H4K20me3 levels in patients with colon cancer. For the first time, this was confirmed through the comparison of cancer versus premalignant state. However, despite its lower levels in patients with colon cancer, the potential of plasma $\mathrm{H} 4 \mathrm{~K} 20 \mathrm{me} 3$ as a diagnostic marker is limited. We found higher amounts of total histone $\mathrm{H} 4$ in plasma of patients with colon cancer, with higher sensitivity and specificity in discriminating colon cancer from other conditions, including normal colonoscopy findings, precancerous polyps and diverticular disease. Further studies with larger sample sizes are warranted to more accurately evaluate the diagnostic impact of this marker in colon cancer, and in other cancer types. Nucleosome-based biomarkers combined with current biomarkers might increase both specificity and sensitivity of tumor detection and may be helpful in individualized precision medicine-based treatments for patients (21).

\section{Conflicts of Interests}

There are no conflicts of interest to declare regarding this study.

\section{Authors' Contributions}

UG and SH conceived of the presented idea and designed the study. EÖ and EEY performed the experiments. MK provided biological materials and clinical data. EÖ and EEY contributed to sample collection. EÖ, EEY and MK contributed to the data analysis. UG wrote the article with support from EÖ, EEY and MK. UG and SH critically revised the article, and gave the final approval of the version to be published. 


\section{Acknowledgements}

This work was supported by the Istanbul University Scientific Projects Unit (Project no: TSA-2017-24494).

\section{References}

1 Ries LAG, Melbert D, Krapcho M, Stinchcomb DG, Howlader N, Horner MJ, Mariotto A, Miller BA, Feuer EJ, Altekruse SF, Lewis DR, Clegg L, Eisner MP, Reichman M and Edwards BK (eds): SEER Cancer Statistics Review, 1975-2005, National Cancer Institute. Bethesda, MD, based on November 2007 SEER data submission, posted to the SEER Web site, 2008. Available at https://seer.cancer.gov/csr/1975_2005/.

2 Siegel RL, Miller KD, Fedewa SA, Ahnen DJ, Meester RGS, Barzi A and Jemal A: Colorectal cancer statistics, 2017. CA Cancer J Clin 67(3): 177-193, 2017. PMID: 28248415. DOI: 10.3322/caac.21395

3 Øines $M$, Helsingen LM, Bretthauer $M$ and Emilsson L: Epidemiology and risk factors of colorectal polyps. Best Pract Res Clin Gastroenterol 31(4): 419-424, 2017. PMID: 28842051. DOI: $10.1016 /$ j.bpg.2017.06.004

4 Chan PW, Ngu JH, Poh Z and Soetikno R: Colorectal cancer screening. Singapore Med J 8(1): 24-28, 2017. PMID: 28111691. DOI: $10.11622 /$ smedj.2017004

5 Zhang J, Cheng Z, Ma Y, He C, Lu Y, Zhao Y, Chang X, Zhang $\mathrm{Y}$, Bai $\mathrm{Y}$ and Cheng N: Effectiveness of screening modalities in colorectal cancer: A network meta-analysis. Clin Colorectal Cancer 16(4): 252-263, 2017. PMID: 28687458. DOI: 10.1016/ j.clcc.2017.03.018

6 Simon K: Colorectal cancer development and advances in screening. Clin Interv Aging 11: 967-976, 2016. PMID: 27486317. DOI: $10.2147 /$ CIA.S 10928

7 Budinska E, Popovici V, Tejpar S, D’Ario G, Lapique N, Sikora KO, Di Narzo AF, Yan P, Hodgson JG, Weinrich S, Bosman F, Roth A and Delorenzi M: Gene expression patterns unveil a new level of molecular heterogeneity in colorectal cancer. J Pathol 231(1): 63-76, 2013. PMID: 23836465. DOI: 10.1002/path.4212

8 Marrugo-Ramírez J, Mir M and Samitier J: Blood based cancer biomarkers in liquid biopsy: A promising non-invasive alternative to tissue biopsy. Int J Mol Sci 19(10), 2018. PMID: 30248975. DOI: 10.3390/ijms 19102877

9 Leszinski G, Gezer U, Siegele B, Stoetzer O and Holdenrieder S: Relevance of histone marks H3K9me3 and H4K20me3 in cancer. Anticancer Res 32(5): 2199-205, 2012. PMID: 22593510.

10 Gezer U, Ustek D, Yörüker EE, Cakiris A, Abaci N, Leszinski G, Dalay N and Holdenrieder S: Characterization of H3K9me3and H4K20me3-associated circulating nucleosomal DNA by high-throughput sequencing in colorectal cancer. Tumour Biol 34(1): 329-336, 2013. PMID: 23086575. DOI: 10.1007/s13277012-0554-5

11 Gezer U, Yörüker EE, Keskin M, Kulle CB, Dharuman Y and Holdenrieder S: Histone methylation marks on circulating nucleosomes as novel blood-based biomarker in colorectal cancer. Int J Mol Sci 16(12): 29654-29662, 2015. PMID: 26690425. DOI: $10.3390 / \mathrm{ijms} 161226180$
12 Bauden M, Pamart D, Ansari D, Herzog M, Eccleston M, Micallef $\mathrm{J}$, Andersson B and Andersson R: Circulating nucleosomes as epigenetic biomarkers in pancreatic cancer. Clin Epigenetics 7: 106, 2015. PMID: 26451166. DOI: 10.1186/s13148-015-0139-4

13 Rahier JF, Druez A, Faugeras L, Martinet JP, Géhénot M, Josseaux E, Herzog M, Micallef J, George F, Delos M, De Ronde T, Badaoui A and D'Hondt L: Circulating nucleosomes as new blood-based biomarkers for detection of colorectal cancer. Clin Epigenetics 9: 53, 2017. PMID: 28515797. DOI: 10.1186/s13148-017-0351-5

14 Fraga MF, Ballestar E, Villar-Garea A, Boix-Chornet M, Espada J, Schotta G, Bonaldi T, Haydon C, Ropero S, Petrie K, Iyer NG, Pérez-Rosado A, Calvo E, Lopez JA, Cano A, Calasanz MJ, Colomer D, Piris MA, Ahn N, Imhof A, Caldas C, Jenuwein T and Esteller M: Loss of acetylation at Lys16 and trimethylation at Lys20 of histone $\mathrm{H} 4$ is a common hallmark of human cancer. Nat Genet 37(4): 391-400, 2005. PMID: 15765097.

15 Tryndyak VP, Kovalchuk O and Pogribny IP: Loss of DNA methylation and histone $\mathrm{H} 4$ lysine 20 trimethylation in human breast cancer cells is associated with aberrant expression of DNA methyltransferase 1, Suv4-20h2 histone methyltransferase and methyl-binding proteins. Cancer Biol Ther 5(1): 65-70, 2006. PMID: 16322686.

16 Van Den Broeck A, Brambilla E, Moro-Sibilot D, Lantuejoul S, Brambilla C, Eymin B, Khochbin S and Gazzeri S: Loss of histone H4K20 trimethylation occurs in preneoplasia and influences prognosis of non-small cell lung cancer. Clin Cancer Res 14(22): 7237-7245, 2008. PMID: 18974389. DOI: 10.1158/ 1078-0432.CCR-08-0869

17 Yokoyama Y, Matsumoto A, Hieda M, Shinchi Y, Ogihara E, Hamada M, Nishioka Y, Kimura H, Yoshidome K, Tsujimoto M and Matsuura N: Loss of histone H4K20 trimethylation predicts poor prognosis in breast cancer and is associated with invasive activity. Breast Cancer Res 16(3): R66, 2014. PMID: 24953066. DOI: $10.1186 /$ bcr3681

18 Rasmussen L, Herzog M, Rømer E, Micallef J, Bulut O, Wilhelmsen M, Christensen IJ and Nielsen HJ: Pre-analytical variables of circulating cell-free nucleosomes containing 5methylcytosine DNA or histone modification H3K9Me3. Scand J Clin Lab Invest 76(6): 448-453, 2016. PMID: 27291394. DOI: 10.1080/00365513.2016.1190862

19 Schwarzenbach H, Hoon DS and Pantel K: Cell-free nucleic acids as biomarkers in cancer patients. Nat Rev Cancer 11(6): 426-437, 2011. PMID: 21562580. DOI: 10.1038/nrc3066

20 Holdenrieder S, Von Pawel J, Nagel D and Stieber P: Long-term stability of circulating nucleosomes in serum. Anticancer Res 30(5): 1613-1615, 2010. PMID: 20592350.

21 McAnena P, Brown JA and Kerin MJ: Circulating nucleosomes and nucleosome modifications as biomarkers in cancer. Cancers (Basel) 9(1), 2017. PMID: 28075351. DOI: 10.3390/cancers 9010005. qdaa

Received May 30, 2019

Revised June 28, 2019

Accepted July 4, 2019 